\title{
ON THE ANALYTIC DISTRIBUTIONS AND FOLIATIONS OF A KÄHLER MANIFOLD
}

\author{
IZU VAISMAN
}

\begin{abstract}
A Frobenius-type condition involving $d_{\bar{z}}$ is proved for the analyticity of a distribution on a complex analytic manifold. As a consequence, an invariant condition for the analyticity of a distribution on a Kähler manifold is derived and used to establish the local reducibility of some foliate Kähler manifolds with bundle-like metric.
\end{abstract}

The aim of this note is to derive invariant conditions for a distribution of a Kähler manifold to be analytic. These conditions will then be used in the study of some questions concerning complex analytic foliations on Kähler manifolds which, until now, were described only by the use of local coordinates [7].

1. Let $V$ be a complex analytic manifold of complex dimension $n$ and $\left\{z^{i}\right\}(i=1, \ldots, n)$-local complex coordinates on $V$. A complex $m$-dimensional distribution $D$ on $V(1 \leqslant m \leqslant n)$ is a differentiable subbundle of the complex tangent bundle $T(V)$, with $m$-dimensional fibres. If we identify $T(V)$ with the bundle which is locally generated by $\left\{\partial / \partial z^{i}\right\}$, it is obvious that the above distribution $D$ is defined locally by

$$
\omega^{a}=\alpha_{i}^{a} d z^{i}=0 \quad(a=1, \ldots, n-m)
$$

where $\alpha_{i}^{a}$ are complex valued differentiable functions on $V$ and $\operatorname{rank}\left(\alpha_{i}^{a}\right)$ $=n-m$. Then, the distribution $D$ is said to be analytic if the system (1.1) is equivalent to a similar system with complex analytic coefficients $\alpha_{i}^{a}$. This condition is obviously independent of the choice of the local coordinates $z^{i}$.

A first analyticity condition which can be easily established is the following Frobenius-type theorem:

1. THEOREM. The distribution $D$ above is analytic iff

$$
d_{\bar{z}} \omega^{a}=0 \quad\left(\operatorname{modulo} \omega^{a}\right),
$$

i.e.

$$
d_{\bar{z}} \omega^{a}=\pi_{b}^{a} \wedge \omega^{b} \quad(a, b=1, \ldots, n-m),
$$

where $\pi_{b}^{a}$ are some locally defined differential forms of type $(0,1)$.

In fact, let (1.1) define an analytic distribution on $V$. Then we must have

Received by the editors December 25, 1974 and, in revised form, November 17, 1975.

AMS (MOS) subject classifications (1970). Primary 53C55, 57D30.

Key words and phrases. Analytic distribution, Kähler-Reinhart space. 
$\omega^{a}=\lambda_{b}^{a} \varpi^{b}$ where $\varpi^{b}$ are analytical 1 -forms and $\operatorname{det}\left(\lambda_{b}^{a}\right) \neq 0$, whence (1.3) follows by a simple computation.

To prove the converse, suppose that (1.3) is satisfied. Then we remark first that $(1.3)$ is also satisfied for any equivalent system $\varpi^{a}=\mu_{b}^{a} \omega^{b}\left(\operatorname{det}\left(\mu_{b}^{a}\right) \neq 0\right)$, which follows by a straightforward computation. Particularly, this is true for the (obviously existing) equivalent system of the form

$$
\theta^{a}=d z^{a}+t_{u}^{a} d z^{u}=0 \quad(a=1, \ldots, n-m ; u=n-m+1, \ldots, n) .
$$

Hence, we have

$$
d_{\bar{z}} \theta^{a}=\pi_{b}^{a} \wedge \theta^{b}
$$

for some forms $\pi_{b}^{a}=\pi_{b c}^{a} d \bar{z}^{c}+\pi_{b u}^{a} d \bar{z}^{u}$, and the identification of the parts of the same type gives $\pi_{b}^{a}=0$. It follows that $\theta^{a}$ have analytic coefficients, which obviously proves our theorem.

Remark. Theorem 1 remains valid if $D$ is defined by a system similar to (1.1) but where $a=1, \ldots, s>n-m$ and rank $\left(\alpha_{i}^{a}\right)=n-m$. In fact, the result follows easily by using Theorem 1 for a subsystem of independent equations.

It is well known that the complex distribution $D$ can also be defined as $D=\operatorname{im} \lambda$, where $\lambda$ is a projector of $T(V)$, i.e. $\lambda: T(V) \rightarrow T(V)$ is a bundle homomorphism such that

$$
\lambda^{2}=\lambda
$$

Then, if

$$
\kappa=1-\lambda
$$

$\kappa$ is also a projector and one has

$$
\kappa^{2}=\kappa, \quad \kappa \lambda=\lambda \kappa=0, \quad D=\operatorname{ker} \kappa .
$$

In this situation, im $\kappa=D^{\prime}$ is a supplementary distribution of $D$.

Now we can give a second condition of analyticity:

2. Theorem. The complex distribution $D$ above is analytic iff, for any local analytic vector fields $v, w$ on $V$, one has

$$
\kappa([\bar{w}, \lambda v])=0,
$$

where the bar means complex conjugate and the bracket is formally defined on $T(V)$ identified with the bundle generated locally by $\left\{\partial / \partial z^{i}\right\}$.

To prove this theorem, let us denote by $\left(\lambda_{j}^{i}\right)$ and $\left(\kappa_{j}^{i}\right)$ the matrices which express $\lambda$ and $\kappa$ with respect to the bases $\left\{\partial / \partial z^{i}\right\}(i, j=1, \ldots, n)$. Then it is easy to see that the local equations (1.1) of $D$ are

$$
\kappa_{j}^{i} d z^{j}=0 .
$$

They are not all independent, but we can use the remark which follows Theorem 1, hence we can use the analyticity condition of Theorem 1. This condition is 


$$
\frac{\partial \kappa_{j}^{i}}{\partial \bar{z}^{k}} d \bar{z}^{k} \wedge d z^{j}=0 \quad\left(\text { modulo } \kappa_{j}^{i} d z^{j}=0\right) .
$$

But, from (1.7) one gets that $\kappa_{j}^{i} d z^{j}=0$ is equivalent to $d z^{j}=\lambda_{i}^{j} d z^{i}$ so that the previous condition becomes

$$
\lambda_{h}^{j} \partial \kappa_{j}^{i} / \partial \bar{z}^{k}=0
$$

or, equivalently, in view of (1.6)

$$
\lambda_{h}^{j} \partial \lambda_{j}^{i} / \partial \bar{z}^{k}=0,
$$

or, still equivalently, in view of $\kappa \lambda=0$,

$$
\kappa_{j}^{i} \partial \lambda_{h}^{j} / \partial \bar{z}^{k}=0 \quad(i, j, h, k=1, \ldots, n) .
$$

Now, the equivalence of (1.12) and (1.8) is a matter of straightforward calculations which we omit here. This proves the theorem.

Let us consider now the usual real structure of $T(V)$. Then the complex distribution $D$ is also a real subbundle of $T(V)$, hence it is defined as the image of a real projector

$$
P: T(V) \rightarrow T(V)
$$

with the supplementary projector $Q=1-P$ which defines $D^{\prime}$. We have

$$
P^{2}=P, \quad Q^{2}=Q, \quad P Q=Q P=0
$$

and $\lambda$ is the complex representation of $P, \kappa$ is the complex representation of $Q$. It is clear that the existence of these complex representations is equivalent to

$$
P J=J P
$$

and, consequently, to

$$
Q J=J Q,
$$

where $J$ denotes the complex structure of $T(V)$ and is such that $J^{2}=-1$.

I.e., $D$ can be defined by the real projector $P$ which satisfies (1.14) and (1.15). For this representation of $D$ we shall prove the following form of the analyticity condition.

3. THEOREM. The distribution $D$ above is analytic iff, for any real vector field $X$ on $V$, one has

$$
Q \circ\left(\nabla_{J X} P-\nabla_{X} P \circ J\right)=0,
$$

where $\nabla$ is an arbitrary hermitian connection on $V$.

Let us consider on $V$ the local differentiable complex coordinates $\left(z^{i}, \bar{z}^{i}\right)$ so that the complexification $T^{c}(V)$ has the bases $\left\{\partial / \partial z^{i}, \partial / \partial \bar{z}^{i}\right\}$ and denote by $i, j, \ldots$ and $\bar{i}, \bar{j}, \ldots$ the indices of the components of the complex tensors on $V$ with respect to these bases [8]. 
Then, the linear extensions of $P$ and $Q$ to $T^{c}(V)$ have the components

$$
\begin{array}{lll}
P_{j}^{i}=\lambda_{j}^{i}, & P_{j}^{\bar{i}}=\bar{\lambda}_{j}^{i}, & P_{j}^{\bar{i}}=P_{\bar{j}}^{i}=0, \\
Q_{j}^{i}=\kappa_{j}^{i}, & Q_{j}^{i}=\bar{\kappa}_{j}^{i}, & Q_{j}^{\bar{i}}=Q_{j}^{i}=0,
\end{array}
$$

and from (1.12) we get that $D$ is analytic iff

$$
Q_{j}^{i} \frac{\partial P_{h}^{j}}{\partial \bar{z}^{k}}=0, \quad Q_{j}^{i} \frac{\partial P_{\bar{h}}^{\bar{j}}}{\partial z^{k}}=0 .
$$

Introduce an arbitrary hermitian metric on $V$ and let $\nabla$ be the corresponding hermitian connection. Since this connection is of type $(1,0)$ (see for instance [6]), it is easy to see that (1.18) is equivalent to

$$
Q_{j}^{i} \nabla_{\bar{k}} P_{h}^{j}=Q_{j}^{i} \nabla_{k} P \frac{\bar{j}}{h}=0 .
$$

Now, we see that (1.19) means that $Q_{\beta}^{\alpha} \nabla_{\gamma} P_{\sigma}^{\beta}$, where $\alpha, \beta, \gamma, \sigma=1, \ldots, 2 n$ denote indices for tensor components with respect to arbitrary differentiable coordinate systems on $V$, is a pure tensor on $V$, which can be equivalently expressed, using the conditions of [8], by

$$
Q_{\beta}^{\alpha}\left(J_{\tau}^{\sigma} \nabla_{\sigma} P_{\nu}^{\beta}-J_{\nu}^{\sigma} \nabla_{\tau} P_{\sigma}^{\beta}\right)=0 .
$$

Finally, the equivalence of (1.20) and (1.17) will be derived by a straightforward calculation, which ends the proof of the theorem.

Remark. From Theorem 3 , one deduces that $D=\operatorname{im} P$ and $D^{\prime}=\operatorname{im} Q$ are both analytic iff

$$
\nabla_{J X} P-\nabla_{X} P \circ J=0
$$

for any vector field $X$ on $V$. This follows from (1.17) and from the similar condition for $D^{\prime}$, using $P+Q=1$. (1.21) is just the condition that $P$ (and also $Q)$ be an analytic tensor [8].

In the particular case of a Kähler manifold we shall take for $\nabla$ in (1.17) the Levi-Civita connection of the Kähler metric and one obtains a simpler form of the analyticity condition.

4. TheOREM. The distribution $D=\operatorname{im} P$ defined on the Kähler manifold $V$ is analytic iff

$$
Q([J X, P Y]-J[X, P Y])=0
$$

for any vector fields $X, Y$ on $V$.

The proof consists of a lengthy but not difficult computation which shows that, in view of the known properties of $P$ and $Q$ and of the properties $\nabla J=0, T(\nabla)=0$ (i.e. $\nabla$ has no torsion), the condition (1.20) (or, which is the same thing (1.17)) is equivalent to (1.22). We omit here this computation. In this proof the condition that $V$ be a Kähler manifold is essential.

REMARK. The condition of Theorem 4 can also be expressed in a form which does not imply the explicit use of $P$ and $Q$. In fact, this condition is obviously 
equivalent to: the distribution $D$ defined on the Kähler manifold $V$ is analytic iff for any vector field $X$ on $V$ and any vector field $Y$ of $D$, the vector field $[J X, Y]-J[X, Y]$ is in $D$.

2. In this section, we shall use the condition of Theorem 4 to establish some results on analytic foliations of a Kähler manifold.

Let $V$ be a Kähler $n$-dimensional manifold with metric $g$ and let $D$ be an $m$ dimensional complex analytic distribution on $V . D$ is said to define a complex analytic foliation on $V$ if it is also integrable i.e. an involutive distribution. Then $(V, g, D)$ is said to be a foliate Kähler manifold and the maximal connected integral manifolds of $D$ are the leaves of the foliation. Since the complex structure of $D$ is induced by the complex structure $J$ of $V$, it is clear that the leaves of $D$ are complex analytic submanifolds of $V$.

As in $\S 1, D$ can be defined by a projector $P$ and it is convenient to take for $P$ the orthogonal projection with respect to $g$. It is easy to see that this is equivalent with the condition

$$
g(P X, Q Y)=0
$$

for any vector fields $X, Y$ on $V$. (Here, we put again, of course, $Q=1-P$.)

It follows that $(V, g, D)$ is a foliate Kähler manifold if and only if the following relations hold good:

$$
\begin{array}{crrr}
J^{2}=-1, & g(J X, J Y) & =g(X, Y), & \nabla_{X} J=0, \\
P^{2}=P, & P J=J P, & g(P X, Q Y)=0, \\
Q([J X, P Y]-J[X, P Y])=0, & Q[P X, P Y]=0,
\end{array}
$$

where $Q=1-P, \nabla$ is the Levi-Civita connection of $g$, and $X, Y$ are arbitrary vector fields on $V$. Here, the first relations express that $g$ is a Kähler metric and the last condition expresses the integrability of $D$. An alternative description of such manifolds, using local complex coordinates can be found in [7].

An important particular case is obtained if one asks the metric $g$ to be bundle-like in the sense of Reinhart [4]. If one uses the characterization $\left(\nabla_{P X}^{(2)} g\right)(Q Y, Q Z)=0$ of such metrics, where $\nabla^{(2)}$ is the so-called second connection [5], [6], the following condition is obtained:

$$
\begin{gathered}
E(X, Y, Z)=(P X) g(Q Y, Q Z)-g([P X, Q Y], Q Z) \\
-g([P X, Q Z], Q Y)=0,
\end{gathered}
$$

or, after some simple transformations based on (2.2),

$$
(P X) g(Y, Q Z)-g([P X, Y], Q Z)-g([P X, Z], Q Y)=0 .
$$

A manifold $V$ endowed with a structure characterized by (2.2) and (2.3) will be called a Kähler-Reinhart space and in the sequel we shall refer to such spaces.

As a first consequence of the formulas (2.2), (2.3), we can prove the following result, obtained by calculations with local coordinates in [7]:

5. THEOREM. A foliate Kähler manifold $(V, g, D)$ is a Kähler-Reinhart space iff 
the supplementary orthogonal distribution $D^{\prime}$ is involutive.

In fact, let

$$
\Phi(X, Y)=g(X, J Y)
$$

be the fundamental form of the Kähler metric $g$, which, as it is well known, is closed. Then, starting with $d \Phi(Q X, Q Y, P Z)=0$ and calculating with (2.2) one gets, using definition (2.3) of $E$,

$$
g([Q X, Q Y], P J Z)=E(Z, X, J Y)
$$

which obviously yields the announced result.

ReMARK. From the previous theorem it follows that a Kähler-Reinhart space is locally diffeomorphic with a direct product, but it is not necessarily holomorphic or isometric with this direct product.

Moreover, one has the following result which is also a consequence of some more general results of Reinhart [4].

6. COROLlaRY. In a Kähler-Reinhart space, the leaves of the supplementary foliation $D^{\prime}$ are totally geodesic submanifolds.

In fact, the second fundamental form of a leaf of $D^{\prime}$ is given by $g\left(P X, \nabla_{Q Y} Q Z\right)$, and using the usual expression of the Levi-Civita connection, the condition $E=0$, and the involutivity of $D^{\prime}$, it follows easily that this second fundamental form vanishes.

Deeper results about the structure of a Kähler-Reinhart space can be obtained if supplementary conditions are imposed. Thus, we have

7. THEOREM. If $(V, g, D)$ is a Kähler-Reinhart space and if the leaves of $D$ are totally geodesic submanifolds of $V$, then $V$ is a reducible Kähler manifold.

In fact, the leaves of $D$ are totally geodesic submanifolds iff $g\left(Q X, \nabla_{P Y} P Z\right)$ $=0$, which, using the expression of the Levi-Civita connection, reduces to

$$
(Q X) g(P Z, P Y)-g(P Z,[Q X, P Y])-g(P Y,[Q X, P Z])=0,
$$

i.e. just the condition for $g$ to be a bundle-like metric with respect to $D^{\prime}$.

Next, if one starts with the relation $d \Phi(P X, P Y, Q Z)=0$ written in the developed form, and if one uses (2.6), it follows that this relation implies $g([J P Y, Q Z]-J[P Y, Q Z], P X)=0$, which is clearly just the condition for $D^{\prime}$ to be analytic.

Hence $D$ and $D^{\prime}$ are both analytic and have the bundle-like metric $g$ which proves the announced theorem.

In order to give another result, we recall that a generally local vector field $X$ on $V$ is foliate if the corresponding local one-parameter transformation group sends locally leaves to leaves and that this is equivalent to $Q[X, P Y]$ $=0$ for any $Y$ [5], [6]. If, moreover, these are conformal transformations for the induced metric of the leaves, $X$ is called a foliate-conformal infinitesimal transformation. Now, if all the foliate local and global vector fields of the form $Q X$ are foliate-conformal infinitesimal transformations, ${ }^{1}$ we shall say that $V$ has conformal leaves [3] and we shall prove

${ }^{1}$ The existence of such local vector fields becomes obvious by using local coordinates. 
8. TheOREM. A Kähler-Reinhart space with conformal leaves is a reducible Kähler manifold.

In fact, it is clear that the condition for $V$ to have conformal leaves is

$$
\left(L_{Q X} g\right)(P Y, P Z)=\rho g(P Y, P Z),
$$

where $L$ denotes the Lie derivative, $Y, Z$ are arbitrary vector fields and $Q X$ is a foliate field on $V$.

But $\left(L_{Q X} g\right)(P Y, P Z)$ is just the left-hand side of (2.6), whence it follows easily that (2.7) is a "pointwise" condition, hence it is valid for arbitrary vector fields $X, Y, Z$ on $V$. In fact, let $Q X$ be arbitrary and $M \in V$. Imbed $Q X(M)$ in a local foliate field and write (2.7) for this field. This proves the previous assertion.

Now, calculating as in the proof of Theorem 7, (2.7) is seen to imply

$$
g([P Z, Q X]+J[J P Z, Q X], P Y)=\rho g(P Y, P Z) .
$$

If we replace $P Y, P Z$, respectively, by $J P Y, J P Z$, the right-hand side does not change so that the left-hand side does not change either. But this means

$$
\begin{aligned}
g([P Z, Q X]+J[J P Z, Q X], P Y) & =g([J P Z, Q X]-J[P Z, Q X], J P Y) \\
& =-g([P Z, Q X]+J[J P Z, Q X], P Y),
\end{aligned}
$$

and it follows that

$$
g([P Z, Q X]+J[J P Z, Q X], P Y)=\rho g(P Y, P Z)=0,
$$

which gives $\rho=0$.

Hence, the leaves are locally isometric and $D^{\prime}$ is analytic too. Then the announced theorem follows just like Theorem 7.

Again let $V$ be a Kähler-Reinhart space. This space is said to have affinely equal leaves if every local foliate vector field of the form $Q X$ is an infinitesimal affine collineation between the leaves endowed with their Levi-Civita connection. Then we have

9. THEOREM. A Kähler-Reinhart space with affinely equal leaves is affinely reducible. If $D^{\prime}$ has a simply connected leaf $L$, then all the nearby leaves are also simply connected and globally isometric to $L$.

In fact, from the formulas for the second connection $\nabla^{(2)}[6]$ it follows that the Levi-Civita connection of the leaves is induced by $\nabla^{(2)}$. Hence, just like for the usual characterization of an infinitesimal affine collineation [2], one sees that the given space has affinely equal leaves iff for any local foliate field $Q X$ and any vector fields $Y, Z$ the following relation holds:

$$
L_{Q X} \nabla_{P Y}^{(2)} P Z-\nabla_{P Y}^{(2)} L_{Q X} P Z-\nabla_{[Q X, P Y]}^{(2)} P Z=0 .
$$

Using again the formulas of [6] and the fact that $Q X$ is foliate, i.e. $Q[Q X, P Y]=0$, we get the equivalent condition

$$
R_{\nabla^{(2)}}(Q X, P Y)(P Z)=0,
$$

where $R$ denotes the curvature operator. 
Now, since (2.9) depends only on the value of $Q X$ at each point, it follows, like for (2.7), that (2.9) is true for arbitrary vector fields $X, Y, Z$.

Next, from the local expressions of the second connection and of its curvature as given in [5], it follows that (2.9) implies the affine reducibility of $V$ with respect to $\nabla^{(2)}$ [1]. (It is important that in the cited local expressions the coefficients $t_{a}^{u}$ of [5] may be considered zero because $D^{\prime}$ is integrable.)

As for the second part of the theorem, we can prove it by a classical technique of differential topology. In fact, if $L$ is a simply connected leaf of $D^{\prime}$, one gets that the parallel translation of a vector $P X$ along the paths of $L$, with respect to $\nabla^{(2)}$, does not depend on the path. So, we are able to construct a vector field $P X$ along $L$. Next, consider the geodesics of $\nabla^{(2)}$ which start from the points of $L$ and are tangent to this vector field $P X$, and take the tangent vectors of these geodesics. It is not difficult to see that we get in this manner a vector field $P X$ defined in a neighbourhood of $L$ and which defines a local one-parameter group of isometries between the leaves of $D^{\prime}$. Clearly, the assertion of the theorem follows. The necessary calculations are based on the local formulas of [5].

REMARK. The Kählerian nature of the metric was used in the previous theorem only by the fact that $D^{\prime}$ is integrable. Hence, Theorem 9 holds, more generally, for a Riemann manifold carrying two orthogonal foliations $D, D^{\prime}$ such that $D$ has bundle-like metric and affinely equal leaves.

\section{REFERENCES}

1. S. Kashiwabara, On the reducibility of an affinely connected manifold, Tôhoku Math. J. (2) 8 (1956), 13-28. MR 18, 332.

2. S. Kobayashi and K. Nomizu, Foundations of differential geometry. Vol. I, Interscience, New York, 1963. MR 27 \#2945.

3. Y. Mutō, On some properties of a fibred Riemannian manifold, Sci. Rep. Yokohama Nat. Univ. Sect. I 1 (1952), 1-14. MR 15, 827.

4. B. L. Reinhart, Foliated manifolds with bundle-like metrics, Ann. of Math. (2) 69 (1959), 119-132. MR 21 \#6004.

5. I. Vaisman, Variétés riemanniennes feuilletées, Czechoslovak Math. J. 21 (96) (1971), 46-75. MR 44 \#476.

6. Cohomology and differential forms, Dekker, New York, 1973. MR 49 \#6095.

7. - From the geometry of Hermitian foliate manifolds, Bull. Math. Soc. Sci. Math. R. S. Roumanie 17 (1973), 71-100.

8. K. Yano, Differential geometry on complex and almost complex spaces, Internat. Ser. of Monographs in Pure and Appl. Math., vol. 49, Macmillan, New York, 1965. MR 32 \#635.

Seminarul Matematic, Universitate Iasi, IASI, RomÂnia 